\title{
Regions of Exponential Stability for LTI Systems on Nonuniform Discrete Domains
}

\author{
John M. Davis \\ Department of Mathematics \\ Baylor University \\ Waco, TX 76798 \\ Email: John_M_Davis@baylor.edu
}

\author{
Ian A. Gravagne and Robert J. Marks II \\ Department of Electrical and Computer Engineering \\ Baylor University \\ Waco, TX 76798 \\ Email: Ian_Gravagne@baylor.edu, \\ Robert_Marks@baylor.edu
}

\author{
Billy J. Jackson \\ Department of Mathematics \\ Saint Xavier University \\ Chicago, IL 60655 \\ Email: bjackson@sxu.edu
}

\begin{abstract}
For LTI systems on a class of nonuniform discrete domains, we establish a region in the complex plane for which pole placement is a necessary and sufficient condition for exponential stability of solutions of the system. We study the interesting geometry of this region, comparing and contrasting it with the standard geometry of the regions of exponential stability for ODE systems on $\mathbb{R}$ and finite difference/recursive equations on $\mathbb{Z}$. This work connects other results in the literature on the topic and explains the connection geometrically using time scales theory.
\end{abstract}

Index Terms-exponential stability, pole placement, time scales

\section{ExPonEntial StABILITY ON $\mathbb{R}$ AND $\mathbb{Z}$}

Let $A \in \mathbb{R}^{n \times n}$. A basic result concerning the continuous, linear time invariant (LTI) system

$$
\dot{x}(t)=A x(t),
$$

is that solutions are exponentially stable if and only if $\operatorname{spec}(A) \subset \mathbb{C}^{-}$.

A similar basic result for discrete LTI systems

$$
x_{n+1}=\tilde{A} x_{n},
$$

is that solutions are exponentially stable if and only if $\operatorname{spec}(A) \subset\{z \in \mathbb{C}:|z|<1\}$. An equivalent reformulation is that solutions of

$$
\Delta x\left(t_{n}\right)=A x\left(t_{n}\right), \quad A:=\tilde{A}-I,
$$

are exponentially stable if and only if $\operatorname{spec}(A) \subset\{z \in \mathbb{C}: \mid 1+$ $z \mid<1\}$. Here, $\Delta$ denotes the forward difference operator. For reasons that will soon become apparent, we will use (I.3) rather than (I.2) as the canonical discrete LTI system throughout this paper.

Thus, the regions of exponential stability for (I.1) and (I.3) are quite straightforward. This simple geometry is exploited frequently in pole placement arguments for exponential stability . In this paper, we explore the following question: What is the geometry of the region of exponential stability for an LTI system defined on a nonuniform discrete domain?

\section{EXPONENTIAL Stability OF NONUNiform Discrete SYSTEMS}

This question can be efficiently handled using time scales theory [5]; see the Appendix for a brief overview. Let $\mathbb{T}$ be a nonuniform, discrete time scale that is unbounded above, and consider the LTI system

$$
x^{\Delta}(t)=A x(t),
$$

or its equivalent recursive form

$$
x_{n+1}=\left(I+A \mu_{n}\right) x_{n} .
$$

Definition II.1. [25] For $t, t_{0} \in \mathbb{T}$ and $x_{0} \in \mathbb{R}^{n}$, the system

$$
x^{\Delta}(t)=A x(t), \quad x\left(t_{0}\right)=x_{0},
$$

is exponentially stable if there exists a constant $\alpha>0$ such that for every $t_{0} \in \mathbb{T}$ there exists a $K \geq 1$ with

$$
\left\|\Phi_{A}\left(t, t_{0}\right)\right\| \leq K e^{-\alpha\left(t-t_{0}\right)} \text { for } t \geq t_{0},
$$

with $K$ being chosen independently of $t_{0}$. Here, $\Phi_{A}\left(t, t_{0}\right)$ denotes the unique solution to (II.3), also called the transition matrix for (II.3); see the Appendix.

The following theorem due to Pötzsche, Siegmund, and Wirth [25] provides a spectral characterization of the region of exponential stability of (II.2) for scalar problems.

Theorem II.1. [25] Let $\mathbb{T}$ be a time scale which is unbounded above. Fix $t_{0} \in \mathbb{T}$ and let $\lambda \in \mathbb{C}$. Then the scalar equation

$$
x^{\Delta}(t)=\lambda x(t), \quad x\left(t_{0}\right)=x_{0},
$$

is exponentially stable if and only if either of the following holds:

(C1) $\limsup _{T \rightarrow \infty} \frac{1}{T-t_{0}} \int_{t_{0}}^{T} \lim _{s \searrow \mu(t)} \frac{\log |1+s \lambda|}{s} \Delta t<0$,

(C2) For every $T \in \mathbb{T}$, there exists a $t \in \mathbb{T}$ with $t>T$ such that $1+\mu(t) \lambda=0$,

where we use the convention $\log 0=-\infty$ in $(\mathrm{C} 1)$.

Definition II.2. [25] Given a time scale $\mathbb{T}$ which is unbounded above, for arbitrary $t_{0} \in \mathbb{T}$, define the sets

$$
\begin{aligned}
& \mathcal{S}_{\mathbb{C}}(\mathbb{T}):=\{\lambda \in \mathbb{C}:(\mathrm{C} 1) \text { holds }\}, \\
& \mathcal{S}_{\mathbb{R}}(\mathbb{T}):=\{\lambda \in \mathbb{R}:(\mathrm{C} 2) \text { holds }\} .
\end{aligned}
$$


Then the set of exponential stability for $\mathbb{T}$ is given by

$$
\mathcal{S}(\mathbb{T}):=\mathcal{S}_{\mathbb{C}}(\mathbb{T}) \cup \mathcal{S}_{\mathbb{R}}(\mathbb{T}) .
$$

Theorem II.1 extends to the time invariant matrix case as follows.

Theorem II.2. [25] Let $\mathbb{T}$ be a time scale that is unbounded above and let $A \in \mathbb{R}^{n \times n}$ be regressive. Then the following hold:

(i) If the system (II.1) is exponentially stable, then $\operatorname{spec}(A) \subset \mathcal{S}_{\mathbb{C}}(\mathbb{T})$.

(ii) If all eigenvalues $\lambda$ of $A$ are uniformly regressive, (i.e., $\exists K$ such that $\left.0<K \leq|1+\mu(t) \lambda|, \forall t \in \mathbb{T}^{\kappa}\right)$ and if $\operatorname{spec}(A) \subset \mathcal{S}_{\mathbb{C}}(\mathbb{T})$, then (II.1) is exponentially stable.

There are situations where the regressivity assumption fails on general time scales [23], but since here we are assuming $\mu(t)>0$ on $\mathbb{T}$, uniform regressivity of (II.1) is an easily verifiable condition. As such, we assume that the LTI systems under discussion are uniformly regressive. In this case, $\mathcal{S}_{\mathbb{R}}(\mathbb{T})=\varnothing$ so we focus on condition (C1) used to define $\mathcal{S}_{\mathbb{C}}(\mathbb{T})$ and understand its geometry.

For dynamical systems on these general time domains $\mathbb{T}$, understanding the structure of $\mathcal{S}$ plays a key role in various aspects of both the control theory and control applications [2], [3], [9], [21]. However, since $\mathcal{S}$ can be difficult to compute on general time scales, other more tractable sufficient conditions for the exponential stability of (II.1) have been explored [25], [14], [10]. For each fixed $t \in \mathbb{T}$, define the (open) Hilger circle $^{1}$ via

$$
\mathcal{H}_{\mu(t)}:=\left\{z \in \mathbb{C}_{\mu}:\left|z+\frac{1}{\mu(t)}\right|<\frac{1}{\mu(t)}\right\} .
$$

When $\mu_{1}<\mu_{2}, \mathcal{H}_{\mu_{2}} \subset \mathcal{H}_{\mu_{1}}$ which leads to the following alternative to Theorem II.2.

Theorem II.3. Let $\mathbb{T}$ be a time scale which is unbounded above. If $0 \leq \mu(t) \leq \mu_{\max }$ for all $t \in \mathbb{T}$, then there is a region $\mathcal{H}_{\min } \subset \mathbb{C}$, corresponding to $\mu_{\max }$ and given by

$$
\mathcal{H}_{\min }:=\left\{z \in \mathbb{C}_{\mu_{\max }}:\left|z+\frac{1}{\mu_{\max }}\right|<\frac{1}{\mu_{\max }}\right\},
$$

such that $\operatorname{spec}(A) \subset \mathcal{H}_{\min }$ is a sufficient (but not necessary) condition for the exponential stability of (II.1).

When $\mathbb{T}=\mathbb{R}$, then $\mu(t) \equiv 0$ and $\mathcal{H}_{\mu(t)} \equiv \mathbb{C}^{-}$; when $\mathbb{T}=\mathbb{Z}$, then $\mu(t) \equiv 1$ and $\mathcal{H}_{\mu(t)} \equiv\{z \in \mathbb{C}:|1+z|<$ $1, z \neq-1\}$. Therefore, in these cases the region described in Theorem II.3 is the familiar region of exponential stability discussed in Section I. Of course, Theorem II.3 applies to much more general LTI systems than just (I.1) or (I.3), but it is important to notice that is subsumes the canonical cases.

Therefore, Theorem II.3 marks progress toward answering the question posed at the end of Section I, because the

\footnotetext{
${ }^{1}$ More appropriately, the Hilger disk, but this abuse of language is established in the literature now. As explained in the Appendix, $\mathbb{C}_{\mu}$ is the punctured complex plane (with the point $z=-1 / \mu$ removed), and ensures uniform regressivity under our assumptions on $\mathbb{T}$.
}

geometry of $\mathcal{H}_{\min }$ is simple. On the other hand, it is admittedly conservative and only provides a sufficient condition for exponential stability. Furthermore, the region $\mathcal{S}_{\mathbb{C}}$ in Theorem II.2 is a globally determined static region whereas the Hilger circle regions in (II.4) and (II.5) are both local and either static (but highly conservative) or dynamic (but insufficient for pole placement argument). We will show that the complete answer lies in the connection between the nice local geometry of the Hilger circles in Theorem II.3 and the stronger global, static characterization of $\mathcal{S}_{\mathbb{C}}$ in Theorem II.2.

Definition II.3. Let $\mathbb{T}$ be a discrete time scale. We say that $m$ is an asymptotic graininess of $\mathbb{T}$ if, for all $t \in \mathbb{T}$ there is a $T \in$ $\mathbb{T}$ with $t<T$ such that $\mu(T)=m$. Let $M_{\infty}=\left\{m_{1}, \ldots, m_{N}\right\}$ be the set of all asymptotic graininesses of $\mathbb{T}$. By the division algorithm, each positive integer $n$ can be written uniquely as $n=q N+r$. Let

$$
s_{n}:= \begin{cases}m_{r}, & r \neq 0, \\ m_{N}, & r=0,\end{cases}
$$

and consider the $N$-periodic sequence $\left\{s_{n}\right\}_{n=1}^{\infty}$. We say $m_{k} \in$ $M_{\infty}$ has weight $d_{k}$ given by

$$
d_{k}:=N^{*} \limsup _{n \rightarrow \infty} \frac{\#\left\{\mu \in s_{n}: \mu=m_{k}\right\}}{n},
$$

where $N^{*}:=1+2+\cdots+N=\frac{1}{2} N(N+1)$. Here, \# denotes the cardinality of a set.

Example II.1. The conditions given in the previous definition are tractable in many cases.

1) When $\mathbb{T}=\mathbb{Z}$, the only asymptotic graininess is $m=1$, which has weight $d=1$.

2) If $\mathbb{T}$ is the time scale with graininesses $\{1,1,2,1,1,2, \ldots\}$, then the asymptotic graininesses are $m_{1}=1$ with weight $d_{1}=2$ and $m_{2}=2$ with weight $d_{2}=1$.

3) Let $n$ be a fixed positive integer. If $\mathbb{T}$ is a $N$-periodic time scale with a base period of graininesses given by

$$
\{1,2,2,3,3,3, \ldots, \underbrace{n, \ldots, n}_{n \text { times }}\}
$$

then each $m_{k}=k$, is an asymptotic graininess with weight $d_{k}=k, k=1, \ldots, n$. Here, $N=\frac{1}{2} n(n+1)$.

4) Suppose $\mu\left(t_{n}\right)$ is determined by the toss of a fair sixsided die. Then the set of asymptotic graininesses is $\{1,2,3,4,5,6\}$ each with weight $d=1$.

We are now in a position to connect Theorems II.2 and II.3. This connection and its geometric explanation are the main contributions of this work.

Theorem II.4. Suppose $\mathbb{T}$ is a discrete time scale with asymptotic graininesses $\mu_{1}, \ldots, \mu_{N}$ each with respective weights $d_{1}, \ldots, d_{N}$. Suppose (II.1) (or its equivalent recursive form (II.2)) is uniformly regressive on $\mathbb{T}$. Then solutions of (II.1) are exponentially stable (in the sense of Definition II.I) if and 
only if

$$
\prod_{k=1}^{N}\left|1+\mu_{k} \lambda\right|^{d_{k}}<1, \quad \forall \lambda \in \operatorname{spec}(A) .
$$

That is, $\mathcal{S}_{\mathbb{C}}$ is the locus of points in the complex plane determined by (II.6).

Proof: Since we are assuming the system in question in uniformly regressive, $\mathcal{S}_{\mathbb{R}}=\varnothing$ so condition $(\mathrm{C} 1)$ is both necessary and sufficient for exponential stability of solutions of (II.1). Thus,

$$
\begin{aligned}
0 & >\limsup _{T \rightarrow \infty} \frac{1}{T-t_{1}} \int_{t_{1}}^{T} \lim _{s \searrow \mu(t)} \frac{\log |1+s \lambda|}{s} \Delta t \\
& =\limsup _{n \rightarrow \infty} \frac{1}{t_{n}-t_{1}} \sum_{k=1}^{n-1} \frac{\log \left|1+\mu\left(t_{k}\right) \lambda\right|}{\mu\left(t_{k}\right)} \mu\left(t_{k}\right) \\
& =\limsup _{n \rightarrow \infty} \frac{1}{t_{n}-t_{1}} \log \prod_{k=1}^{n-1}\left|1+\mu\left(t_{k}\right) \lambda\right|
\end{aligned}
$$

holds (under our assumptions on $\mathbb{T}$ ) if and only if

$$
\prod_{k=1}^{N}\left|1+\mu_{k} \lambda\right|^{d_{k}}<1
$$

holds every $\lambda \in \operatorname{spec}(A)$.

Remark II.5. Several remarks are now in order regarding Theorem II.4.

1) The locus of points in the complex plane determined by (II.6) is in a sense the weighted geometric mean of the individual asymptotic Hilger circle loci. This follows from the observation that left-hand side of each factor in (II.6) is precisely the left-hand side of the locus in (II.4) (up to the weight $d_{k}$ ). Note that $\left|1+m_{k} z\right|<1$ is equivalent to $\left|z+\frac{1}{m_{k}}\right|<\frac{1}{m_{k}}$.

2) This theorem provides a simple, static region of exponential stability for solutions of (II.1) (and therefore fit for traditional pole placement arguments), yet it is made up global information about the time scale (the aggregate of all asymptotic graininesses) that is an "average" of local information about the time scale (the point by point asymptotic Hilger circles).

3) This formulation leads naturally to the notion of asymptotic equivalence classes of time scales, that is, classes of times scales which have equivalent regions of exponential stability. Two time scales are in the same asymptotic equivalence class if they have the same set of asymptotic graininesses with the same respective weights. For example, the periodic (or eventually periodic) time scales with graininess sequence $\{1,1,2,1,1,2, \ldots\}$ or $\{1,2,1,1,2,1, \ldots\}$ or $\{2,1,1,1,2,1,1,1,2, \ldots\}$ would all be in the same asymptotic equivalence class.

4) As the weight of a particular asymptotic graininess $m_{k}$ increases (without bound), $\mathcal{S}_{\mathbb{C}}(\mathbb{T})$ tends toward $\mathcal{S}_{\mathbb{C}}\left(m_{k} \mathbb{Z}\right)$, i.e. a single Hilger circle centered at $-1 / m_{k}$ and passing through the origin.
5) When, for example, $m_{1}$ is fixed and $m_{2}$ varies (each with weight 1 ), one can determine the critical value $m_{2}=m_{2}^{*}$ for which $\mathcal{S}_{\mathbb{C}}$ becomes disconnected. This is simply the $m_{2}$ value at which the saddle point of

$$
f(x, y)=\prod_{k=1}^{2}\left[\left(1+m_{k} x\right)^{2}+\left(m_{k} y\right)^{2}\right],
$$

increases through a height of 1 .

6) As noted in [25] and reconfirmed here, if $\mu \rightarrow \infty$, then all solutions of (II.1) blow up and therefore no exponentially stable solutions exist. This is not a defect in the equation, but rather a pathology of the underlying domain. Of course, this phenomenon never occurs in the canonical ODE or finite difference equation cases.

7) Also noted in [25] and evident here, $\mathcal{S}_{\mathbb{C}}$ is symmetric about the real axis.

8) The approach here is inherently limited by the structure of the tail of the time scale (or our knowledge of the structure). Naturally, some time scales may have infinitely many asymptotic graininesses-or none. For example, $\mu\left(t_{n}\right)$ might be a random variable.

9) The technique here can be extended to time scales with zero graininess, although the details of the analysis are more complicated. We will treat this scenario in a forthcoming paper. The geometry of $\mathcal{S}_{\mathbb{C}}$ in that case is even more interesting.

Figures 1 and 2 demonstrate the geometry of Theorem II.4 and illustrate several of the remarks above.

\section{SUMMARY AND CONCLUSIONS}

To recapitulate, for LTI systems on a class of nonuniform discrete domains, Theorem II.4 establishes a locus of points in the complex plane for which pole placement is both a necessary and sufficient condition for exponential stability of solutions of the associated system. Moreover, the analytic form of (II.6) yields important geometric insight about the region: it is in a sense the weighted geometric mean of the relevant asymptotic Hilger circles. This not only unifies the analogous concepts on $\mathbb{R}$ and $\mathbb{Z}$, but extends them to more general time domains as well.

\section{APPENDIX}

\section{A. What Are Time Scales?}

The theory of time scales springs from the 1988 doctoral dissertation of Stefan Hilger [19] that resulted in his seminal paper [18]. These works aimed to unify various overarching concepts from the (sometimes disparate) theories of discrete and continuous dynamical systems [24], but also to extend these theories to more general classes of dynamical systems. From there, time scales theory advanced fairly quickly, culminating in the excellent introductory text by Bohner and Peterson [4] and the more advanced monograph [5]. A succinct survey on time scales can be found in [1].

A time scale $\mathbb{T}$ is any nonempty, (topologically) closed subset of the real numbers $\mathbb{R}$. Thus time scales can be (but are 

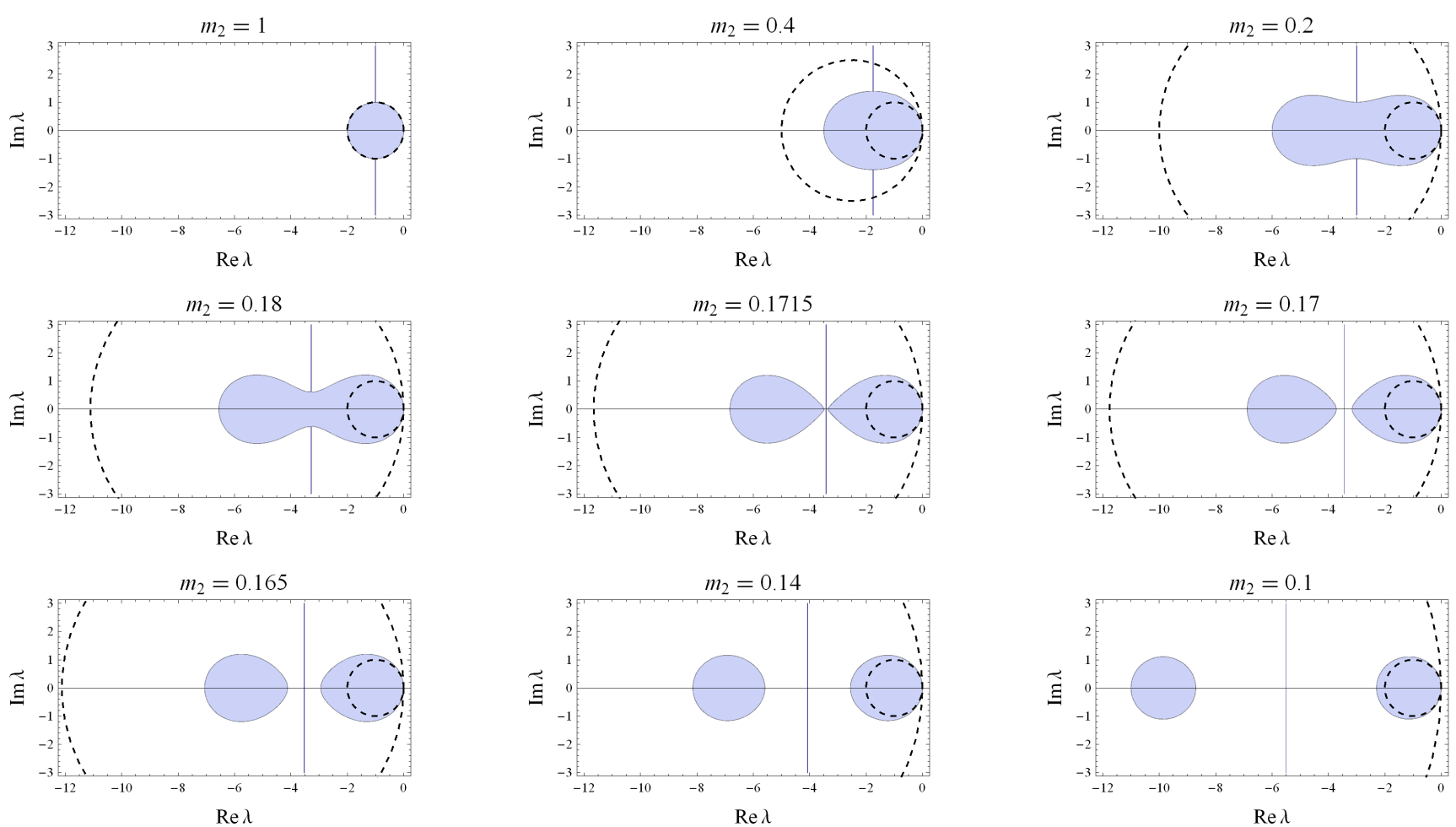

Fig. 1. (Read from left to right, starting from the top row) $\mathbb{T}$ has asymptotic graininesses $m_{1}=1$ and $m_{2}$ (which varies), each with weight 1 . The two corresponding asymptotic Hilger circles are dashed. The region of exponential stability $\mathcal{S}_{\mathbb{C}}$ given by (II.6) is shaded. The vertical line is the arithmetic mean $\bar{m}$ of the centers of the Hilger circles and is an axis of symmetry for $\mathcal{S}_{\mathbb{C}}$ when it lies to the left -2 (i.e. outside the smallest asymptotic Hilger circle). Geometrically, the region of exponential stability can be interpreted as the weighted geometric mean of the asymptotic Hilger circles.
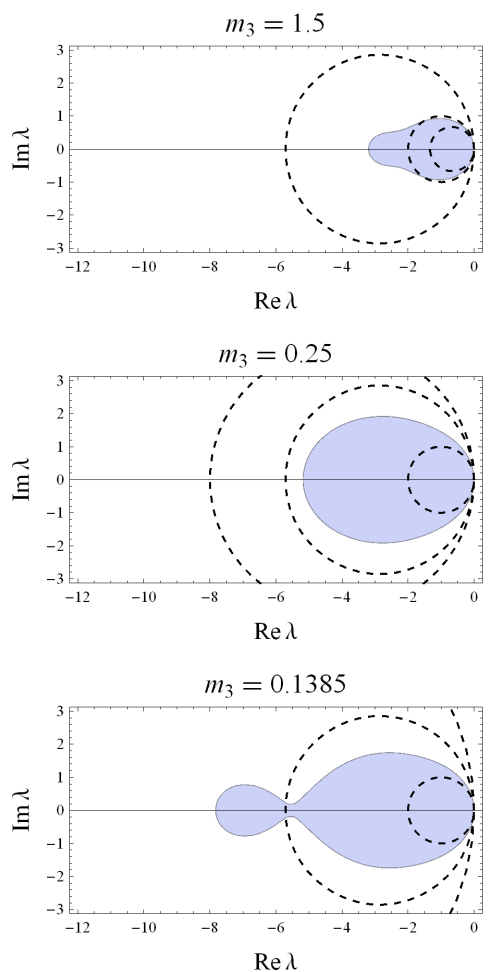
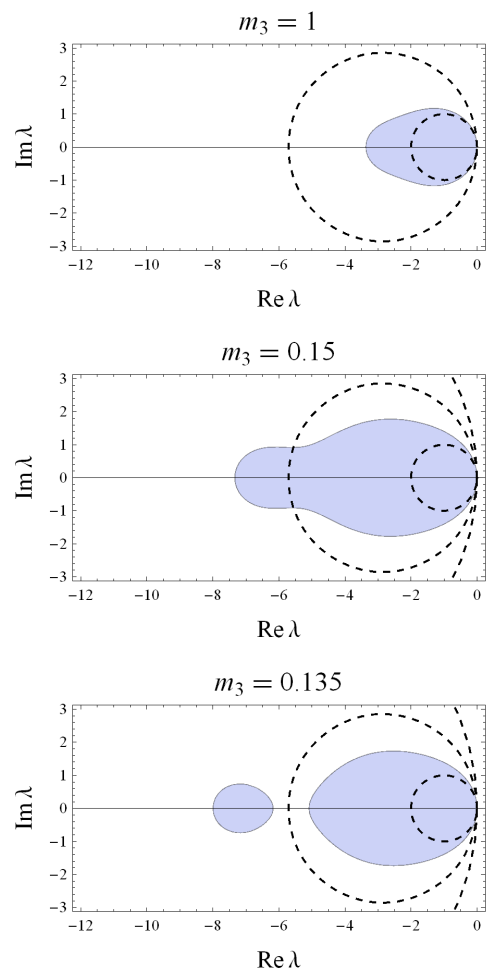
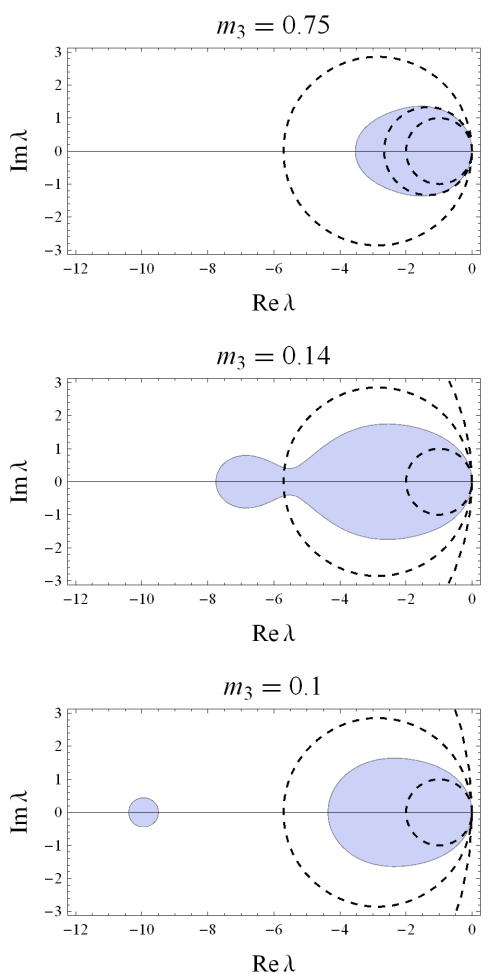

Fig. 2. (Read from left to right, starting from the top row) $\mathbb{T}$ has asymptotic graininesses $m_{1}=1, m_{2}=0.35$, and $m_{3}$ (which varies), each with weight 1. The three corresponding asymptotic Hilger circles are dashed. The region of exponential stability $\mathcal{S}_{\mathbb{C}}$ given by (II.6) is shaded. Geometrically, the region of exponential stability can be interpreted as the weighted geometric mean of the asymptotic Hilger circles. 
TABLE I

CANONICAL TIME SCALES COMPARED TO THE GENERAL CASE.

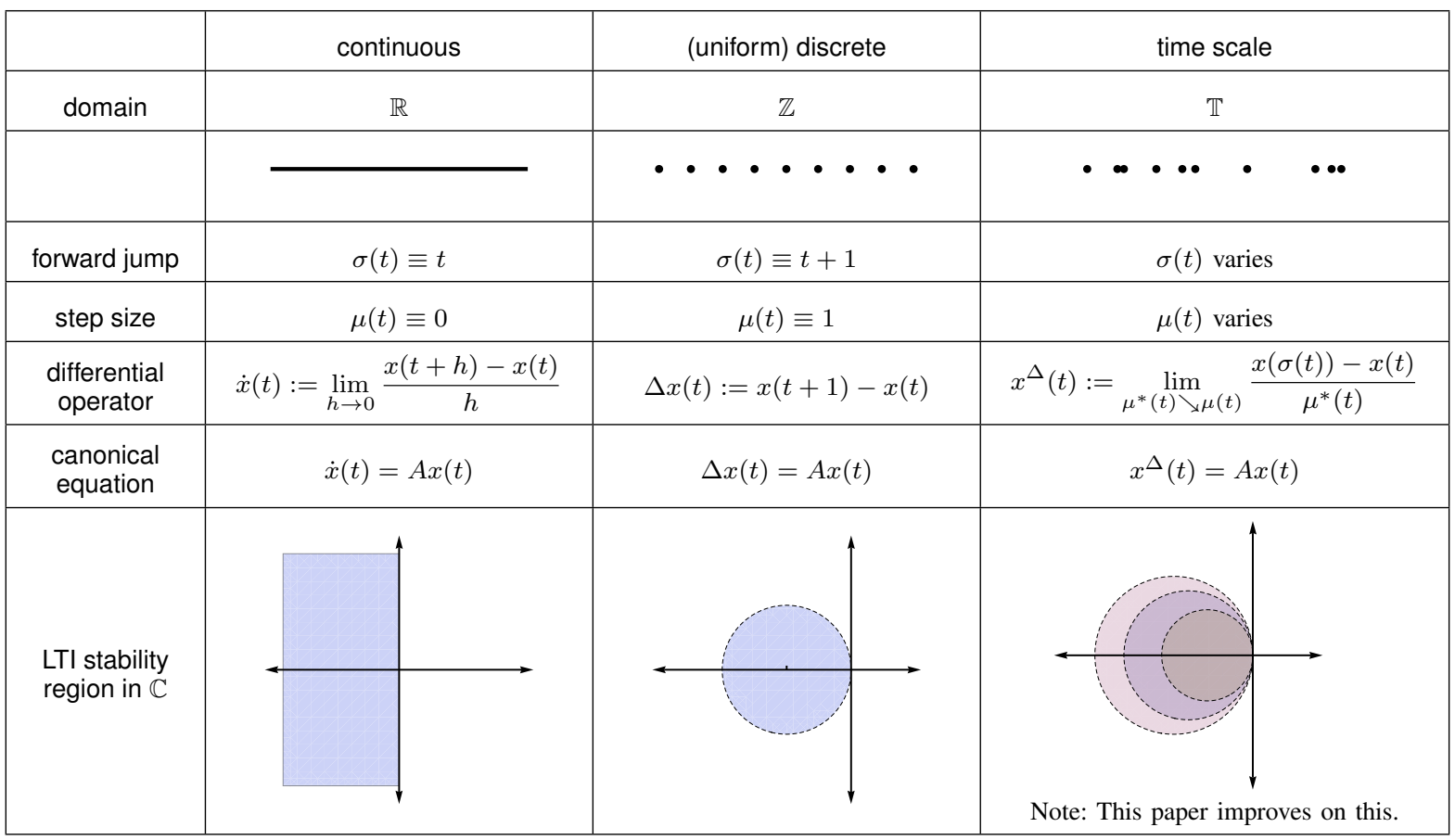

not limited to) any of the usual integer subsets (e.g. $\mathbb{Z}$ or $\mathbb{N}$ ), the entire real line $\mathbb{R}$, or any combination of discrete points unioned with closed intervals. For example, if $q>1$ is fixed, the quantum time scale $\overline{q^{\mathbb{Z}}}$ is defined as

$$
\overline{q^{\mathbb{Z}}}:=\left\{q^{k}: k \in \mathbb{Z}\right\} \cup\{0\} .
$$

The quantum time scale appears throughout the mathematical physics literature, where the dynamical systems of interest are the $q$-difference equations [6], [7], [8]. Another interesting example is the pulse time scale $\mathbb{P}_{a, b}$ formed by a union of closed intervals each of length $a$ and gap $b$ :

$$
\mathbb{P}_{a, b}:=\bigcup_{k}[k(a+b), k(a+b)+a] .
$$

This time scale is used to study duty cycles of various waveforms. Other examples of interesting time scales include any collection of discrete points sampled from a probability distribution, any sequence of partial sums from a series with positive terms, or even the famous Cantor set.

The bulk of engineering systems theory to date rests on two time scales, $\mathbb{R}$ and $\mathbb{Z}$ (or more generally $h \mathbb{Z}$, meaning discrete points separated by distance $h$ ). However, there are occasions when necessity or convenience dictates the use of an alternate time scale. The question of how to approach the study of dynamical systems on time scales then becomes relevant, and in fact the majority of research on time scales so far has focused on expanding and generalizing the vast suite of tools available to the differential and difference equation theorist. We now briefly outline the portions of the time scales theory that are needed for this paper to be as self-contained as is practically possible.

\section{B. The Time Scales Calculus}

The forward jump operator is given by $\sigma(t):=\inf _{s \in \mathbb{T}}\{s>$ $t\}$ and the graininess function $\mu(t)$ by $\mu(t):=\sigma(t)-t$. If $f: \mathbb{T} \rightarrow \mathbb{R}$ is a function, then the composition $f(\sigma(t))$ is often denoted by $f^{\sigma}(t)$.

A point $t \in \mathbb{T}$ is right-scattered if $\sigma(t)>t$ and right dense if $\sigma(t)=t$. A point $t \in \mathbb{T}$ is left-scattered if $\rho(t)<t$ and left dense if $\rho(t)=t$. If $t$ is both left-scattered and right-scattered, we say $t$ is isolated or discrete. If $t$ is both left-dense and rightdense, we say $t$ is dense. The set $\mathbb{T}^{\kappa}$ is defined as follows: if $\mathbb{T}$ has a left-scattered maximum $m$, then $\mathbb{T}^{\kappa}=\mathbb{T}-\{m\}$; otherwise, $\mathbb{T}^{\kappa}=\mathbb{T}$.

For $f: \mathbb{T} \rightarrow \mathbb{R}$ and $t \in \mathbb{T}^{\kappa}$, define $f^{\Delta}(t)$ as the number (when it exists), with the property that, for any $\varepsilon>0$, there exists a neighborhood $U$ of $t$ such that for all $s \in U$,

$$
\left|[f(\sigma(t))-f(s)]-f^{\Delta}(t)[\sigma(t)-s]\right| \leq \epsilon|\sigma(t)-s| .
$$

The function $f^{\Delta}: \mathbb{T}^{\kappa} \rightarrow \mathbb{R}$ is called the delta derivative or the Hilger derivative of $f$ on $\mathbb{T}^{\kappa}$. Equivalently, (A.1) defines the $\Delta$-differential operator via

$$
x^{\Delta}(t):=\lim _{\mu^{*}(t) \searrow \mu(t)} \frac{x(\sigma(t))-x(t)}{\mu^{*}(t)} .
$$

Since the graininess function induces a measure on $\mathbb{T}$, if we consider the Lebesgue integral over $\mathbb{T}$ with respect to the $\mu$ induced measure, $\int_{\mathbb{T}} f(t) d \mu(t)$, then all of the standard results from measure theory are available [17].

A benefit of this general approach is that the realms of differential equations and difference equations can now be viewed as special cases of more general dynamic equations on 
TABLE II

DIFFERENTIAL AND INTEGRAL OPERATORS ON TIME SCALES.

\begin{tabular}{|c|c|c|c|c|}
\hline time scale & differential operator & notes & integral operator & notes \\
\hline $\mathbb{T}$ & $x^{\Delta}(t):=\lim _{\mu^{*}(t) \backslash \mu(t)} \frac{x(\sigma(t))-x(t)}{\mu^{*}(t)}$ & generalized derivative & $\int_{a}^{b} f(t) \Delta t$ & generalized integral \\
\hline $\mathbb{R}$ & $x^{\Delta}(t)=\lim _{h \rightarrow 0} \frac{x(t+h)-x(t)}{h}$ & standard derivative & $\int_{a}^{b} f(t) \Delta t=\int_{a}^{b} f(t) d t$ & standard Lebesgue integral \\
\hline $\mathbb{Z}$ & $x^{\Delta}(t)=\Delta x(t):=x(t+1)-x(t)$ & forward difference & $\int_{a}^{b} f(t) \Delta t=\sum_{t=a}^{b-1} f(t)$ & summation operator \\
\hline$h \mathbb{Z}$ & $x^{\Delta}(t)=\Delta_{h} x(t):=\frac{x(t+h)-x(t)}{h}$ & $h$-forward difference & $\int_{a}^{b} f(t) \Delta t=\sum_{t=a}^{b-h} f(t) h$ & $q$-summation \\
\hline$\overline{q^{\mathbb{Z}}}$ & $x^{\Delta}(t)=\Delta_{q} x(t):=\frac{x(q t)-x(t)}{(q-1) t}$ & $q$-difference & $\int_{a}^{b} f(t) \Delta t=\sum_{t=a}^{b / q} \frac{f(t)}{(q-1) t}$ & $q$-summation \\
\hline $\mathbb{P}_{a, b}$ & $x^{\Delta}(t)=\left\{\begin{array}{lll}\frac{d x}{d t}, \\
\frac{x(t+b)-x(t)}{b}, & \sigma(t)>t\end{array}\right.$ & pulse derivative & $\int_{I} f(t) \Delta t= \begin{cases}\int_{I} f(t) d t, & \sigma(t)=t, \\
f(t) \mu(t), & \sigma(t)>t\end{cases}$ & pulse integral \\
\hline
\end{tabular}

time scales, i.e. equations involving the delta derivative(s) of some unknown function. The upshot here is that the concepts in Tables I and II apply just as readily to any closed subset of the real line as they do on $\mathbb{R}$ or $\mathbb{Z}$. Our goal is to leverage this general framework against wide classes of dynamical and control systems. Progress in this direction has been made in transforms theory [11], [12], [13], [22], control [10], [15], [16], dynamic programming [26], and biological models [20].

The function $p: \mathbb{T} \rightarrow \mathbb{R}$ is regressive if $1+\mu(t) p(t) \neq 0$ for all $t \in \mathbb{T}^{\kappa}$. We define the related sets $\mathcal{R}:=\{p: \mathbb{T} \rightarrow$ $\mathbb{R}: p \in \mathrm{C}_{\mathrm{rd}}(\mathbb{T})$ and $1+\mu(t) p(t) \neq 0$ for all $\left.t \in \mathbb{T}^{\kappa}\right\}$ and $\mathcal{R}^{+}:=\left\{p \in \mathcal{R}: 1+\mu(t) p(t)>0\right.$ for all $\left.t \in \mathbb{T}^{\kappa}\right\}$.

For $p(t) \in \mathcal{R}$, we define the generalized time scale exponential function $e_{p}\left(t, t_{0}\right)$ as the unique solution to the initial value problem $x^{\Delta}(t)=p(t) x(t), x\left(t_{0}\right)=1$, which exists when $p \in \mathcal{R}$. See [5].

Similarly, the unique solution to the matrix initial value problem $X^{\Delta}(t)=A(t) X(t), X\left(t_{0}\right)=I$ is called the transition matrix associated with this system. This solution is denoted by $\Phi_{A}\left(t, t_{0}\right)$ and exists when $A \in \mathcal{R}$. A matrix is regressive if and only if all of its eigenvalues are in $\mathcal{R}$. Equivalently, the matrix $A(t)$ is regressive if and only if $I+\mu(t) A(t)$ is invertible for all $t \in \mathbb{T}^{\kappa}$.

\section{ACKNOWLEDGEMENTS}

This work was supported by National Science Foundation grant CMMI \#0726996.

\section{REFERENCES}

[1] R. Agarwal, M. Bohner, D. O'Regan, and A. Peterson, Dynamic equations on time scales: a survey, Journal of Computational and Applied Mathematics 141 (2002), 1-26.

[2] Z. Bartosiewicz and E. Pawłuszewicz, Realizations of linear control systems on time scales, Control \& Cybernetics 35 (2006).

[3] Z. Bartosiewicz and E. Pawłuszewicz, Realizations of nonlinear control systems on time scales, IEEE Trans. Automatic Control 53 (2008), 571575.

[4] M. Bohner and A. Peterson, Advances in Dynamic Equations on Time Scales, Birkhäuser, Boston, 2003.

[5] M. Bohner and A. Peterson, Dynamic Equations on Time Scales, Birkhäuser, Boston, 2001.

[6] D. Bowman, " $q$-Difference Operators, Orthogonal Polynomials, and Symmetric Expansions" in Mem. Amer. Math. Soc. 159 (2002), 1-56.

[7] R.W. Carroll, Calculus Revisited, Kluwer, Dordrecht, 2002.
[8] P. Cheung and V. Kac, Quantum Calculus, Springer-Verlag, New York, 2002.

[9] J.J. DaCunha, Stability for time varying linear dynamic systems on time scales, J. Comput. Appl. Math. 176 (2005), 381-410.

[10] J.M. Davis, I.A. Gravagne, B.J. Jackson, and R.J. Marks II, Controllability, observability, realizability, and stability of dynamic linear systems, Electronic Journal of Differential Equations 2009 (2009), 1-32.

[11] J.M. Davis, I.A. Gravagne, B.J. Jackson, R.J. Marks II, and A.A. Ramos, The Laplace transform on time scales revisited, J. Math. Anal. Appl. 332 (2007), 1291-1306.

[12] J.M. Davis, I.A. Gravagne, and R.J. Marks II, Bilateral Laplace transforms on time scales: convergence, convolution, and the characterization of stationary stochastic time series, Circuits, Systems, and Signal Processing, 29 (2010), 1141-1165.

[13] J.M. Davis, I.A. Gravagne, and R.J. Marks II, Convergence of unilateral Laplace transforms on time scales, Circuits, Systems, and Signal Processing, 29 (2010), 971-997.

[14] T. Gard and J. Hoffacker, Asymptotic behavior of natural growth on time scales, Dynam. Systems Appl. 12 (2003), 131-147.

[15] I.A. Gravagne, J.M. Davis, J.J. DaCunha, A unified approach to highgain adaptive controllers, Abstract and Applied Analysis, 2009 (2009), $1-13$.

[16] I.A. Gravagne, J.M. Davis, J.J. DaCunha, and R.J. Marks II, Bandwidth reduction for controller area networks using adaptive sampling, Proc. Int. Conf. Robotics and Automation, New Orleans, LA (2004), 52505255.

[17] G. Guseinov, Integration on time scales. J. Math. Anal. Appl. 285 (2003), $107-127$.

[18] S. Hilger, Analysis on measure chains-a unified approach to continuous and discrete calculus, Results Math. 18 (1990), 18-56.

[19] S. Hilger, Ein Maßkettenkalkül mit Anwendung auf Zentrumsmannigfaltigkeiten, Ph.D. thesis, Universität Würzburg, 1988.

[20] J. Hoffacker and B.J. Jackson, A time scale model of interacting transgenic and wild mosquito populations, preprint.

[21] B.J. Jackson, J.M. Davis, I.A. Gravagne, R.J. Marks II, Linear state feedback stabilization on time scales, International Journal of Dynamical Systems and Differential Equations, to appear.

[22] R.J. Marks II, I.A. Gravagne, and J.M. Davis, A generalized Fourier transform and convolution on time scales. J. Math. Anal. Appl. 340 (2008), 901-919.

[23] R.J. Marks II, I.A. Gravagne, J.M. Davis, J.J. DaCunha, Nonregressivity in switched linear circuits and mechanical systems, Math. Comput. Modelling 43 (2006), 1383-1392.

[24] A.N. Michel, L. Hou, and D. Liu, Stability of Dynamical Systems: Continuous, Discontinuous, and Discrete Systems, Birkhäuser, Boston, 2008.

[25] C. Pötzsche, S. Siegmund, and F. Wirth, A spectral characterization of exponential stability for linear time-invariant systems on time scales, Discrete Contin. Dyn. Syst. 9 (2003), 1223-1241.

[26] J. Seiffertt, S. Sanyal, and D.C. Wunsch, Hamilton-Jacobi-Bellman equations and approximate dynamic programming on time scales, IEEE Trans. Systems, Man, and Cybernetics B 38 (2008), 918-923.

[27] http://timescales.org/ 